\title{
Blind Symbol Synchronization Based on Cyclic Prefix for OFDM Systems
}

\author{
Shaodan Ma, Xinyue Pan, Guang-Hua Yang, and Tung-Sang Ng, Fellow, IEEE
}

\begin{abstract}
In this paper, a blind symbol synchronization algorithm is presented for orthogonal frequency-division multiplexing (OFDM) systems, and a new timing function based on the redundancy of the cyclic prefix $(\mathrm{CP})$ is introduced. It proves that the maximum of this function necessarily points to the correct timing offset, irrespective of channel conditions when the signal-to-noise ratio is high. Using the timing function, the timing offset is estimated through a searching algorithm. Channel power profile and channel length information are unnecessary. Simulation results show that the proposed algorithm is robust and outperforms the existing CP-based algorithms, particularly in frequency-selective fading channels.
\end{abstract}

Index Terms-Orthogonal frequency-division multiplexing (OFDM), symbol synchronization.

\section{INTRODUCTION}

$\mathbf{I}$ ORTHOGONAL frequency-division multiplexing (OFDM) systems, synchronization issues are of great importance since synchronization errors might destroy the orthogonality among all subcarriers and, therefore, introduce intercarrier interference (ICI) and intersymbol interference (ISI) [1]-[3]. Generally, synchronization tasks include sampling clock synchronization, symbol synchronization, and carrier frequency synchronization [4]. Sampling clock synchronization mitigates the sampling clock errors due to the mismatch of the crystal oscillators, while carrier frequency synchronization eliminates the carrier frequency offset arising from transceiver oscillator mismatches and/or Doppler shifts. In this paper, symbol synchronization is the focus. The objective is to find the correct starting position [i.e., the fast Fourier transform (FFT) window] of the OFDM symbol for FFT demodulation. It is equivalent to estimate the timing offset between the transmitter and receiver. This is also referred to as timing synchronization. Note that the timing offset is an integer and can be anywhere within an OFDM symbol.

Symbol synchronization may be performed at the receiver using a predesigned preamble [4]-[7]. Although accurate estimation can be achieved, the bandwidth efficiency is inevitably reduced. To eliminate this reduction, algorithms using the

Manuscript received April 1, 2008; revised July 11, 2008. First published August 12, 2008; current version published April 22, 2009. This work was supported by the Hong Kong Research Grants Council under Grant 7160/06E. The review of this paper was coordinated by D. Marabissi.

The authors are with the Department of Electrical and Electronic Engineering, The University of Hong Kong, Pokfulam, Hong Kong (e-mail: sdma@ eee.hku.hk; xypan@eee.hku.hk; ghyang@eee.hku.hk; tsng@eee.hku.hk).

Color versions of one or more of the figures in this paper are available online at http://ieeexplore.iee.org.

Digital Object Identifier 10.1109/TVT.2008.2004031 redundancy introduced by the cyclic prefix $(\mathrm{CP})$ have been proposed [4], [8]-[12]. The most representative one is the maximum likelihood (ML) symbol synchronization algorithm [8]. However, good performance is achieved only under flat-fading channels. When the system is operating under frequencyselective fading channels, the ML algorithm exhibits significant fluctuation in the estimated timing offset. This is because the maximum of the timing function depends on channel conditions and does not necessarily point to the correct timing offset due to ISI under frequency-selective fading channels. Recently, a number of algorithms have been proposed in [9]-[12] to combat the effect of ISI and mitigate the fluctuation. Unfortunately, the double correlation method in [9] works well only when the first path channel response is the strongest. The correlation derivative algorithm in [10] makes use of the property that the correlator output is the sum of a set of triangular functions and is sensitive to the carrier frequency offset, as well as the filter length used [13]. The rank method based on minimum description length (MDL) criterion in [11] is computation intensive. The asymptotic ML algorithm in [12] uses the correlation length equal to the sum of the channel and CP lengths. It is rather impractical since exact knowledge of the channel power profile and channel length is required. The simplified minimum mean square error and maximum correlation algorithms in [12] set the correlation length to twice the $\mathrm{CP}$ length and still include ISI in the timing function, resulting in fluctuation and poor performance.

In this paper, a blind symbol synchronization algorithm is proposed for OFDM systems, and a new timing function is designed based on the redundancy introduced by the CP. Using the proposed timing function, the timing offset is estimated through a 2-D search. It is proved that under a high signalto-noise ratio (SNR) assumption, the maximum of the timing function necessarily points to the correct timing offset, irrespective of channel conditions, even under frequency-selective fading channels. Since the position uncertainty in the maximum point is removed, the fluctuation of the estimated timing offset is mitigated, and the estimation accuracy is thus improved. In addition, there is no restriction on the strength of the first path channel response, and channel information (including channel power profile and channel length) is not required. Computer simulations show that the proposed algorithm achieves an unbiased estimation of the timing offset and that the mean square of the estimation error is significantly reduced compared with the existing CP-based algorithms when the system is operating under frequency-selective fading channels. It is also verified that the proposed algorithm is robust to frequency-selective fading channels. 
The rest of this paper is organized as follows. In Section II, the OFDM system model is introduced. The CP-based blind symbol synchronization algorithm is presented in Section III, and in Section IV, the performance of the proposed algorithm is demonstrated by computer simulations. Finally, Section V draws the conclusion.

\section{SySTEM MODEL}

Consider a baseband discrete-time OFDM system with $N$ subcarriers. The independent identically distributed (i.i.d.) complex signals $d(n), n \in\{0,1, \ldots, N-1\}$, are modulated onto the $N$ subcarriers by means of the inverse discrete Fourier transform (IDFT). The CP with length $N_{\mathrm{cp}}$ is then inserted at the beginning of each OFDM symbol to avoid ISI and preserve the orthogonality among subcarriers. The OFDM symbol $s(n), n \in\left\{0,1, \ldots, N+N_{\mathrm{cp}}-1\right\}$, is finally serially transmitted through a frequency-selective fading channel, which is generally modeled as an $(L+1)$-tap finite-impulse response filter. Here, the channel length $L$ is assumed to be shorter than the CP length $N_{\text {cp }}$.

At the receiver, assuming perfect sampling clock synchronization has been achieved, the received sampled signal can be expressed as

$$
\begin{aligned}
r(n)=e^{j 2 \pi \varepsilon n / N} \sum_{l=0}^{L} h(l) s(n-l-\theta)+w(n) & \\
& n=0,1, \ldots, N+N_{\mathrm{cp}}-1
\end{aligned}
$$

where $\varepsilon$ denotes the carrier frequency offset (which is normalized by the subcarrier spacing) due to the mismatch of the transceiver oscillators and/or the Doppler frequency shift, $\theta$ represents the timing offset to be estimated, $\theta \in\{0,1, \ldots, N+$ $\left.N_{\text {cp }}-1\right\}, h(l)$ is the response of the frequency-selective fading channel, $s(n)$ is the OFDM signal after CP insertion, and $w(n)$ denotes the additive white Gaussian noise (AWGN) with zero mean and variance $\sigma_{n}^{2}$, which is independent of the OFDM signals $s(n)$.

\section{CP-BASED BLIND SyMbOL SYNCHRONIZATION}

Now, consider the correlation between the received sampled signals $r(n)$ and $r(n+N)$. From (1), it can be written as

$$
E\left\{r(n) r^{*}(n+N)\right\}=E\{a(n)+b(n)\}
$$

where

$$
\begin{aligned}
a(n)= & e^{-j 2 \pi \varepsilon}\left(\sum_{l=0}^{L} h(l) s(n-l-\theta)\right) \\
& \times\left(\sum_{l=0}^{L} h^{*}(l) s^{*}(n+N-l-\theta)\right)
\end{aligned}
$$

$$
\begin{aligned}
b(n)= & e^{j 2 \pi \varepsilon n / N}\left(\sum_{l=0}^{L} h(l) s(n-l-\theta)\right) w^{*}(n+N) \\
& +e^{-j 2 \pi \varepsilon(n+N) / N}\left(\sum_{l=0}^{L} h^{*}(l) s^{*}(n+N-l-\theta)\right) w(n) \\
& +w(n) w^{*}(n+N) .
\end{aligned}
$$

Since the zero mean AWGN noise $w(n)$ is independent of the OFDM signals, $E\{b(n)\}=0$. On the other hand, the OFDM signal $s(n)$ is the IDFT of the i.i.d. signals $d(n)$, and the signals in the CP $\left\{s(0), s(1), \ldots, s\left(N_{\mathrm{cp}}-1\right)\right\}$ are a copy of the last $N_{\text {cp }}$ samples in the body of the OFDM symbol. The correlation of the OFDM signal $s(n)$ therefore satisfies

$$
\begin{aligned}
& E\left\{s\left(n_{1}\right) s^{*}\left(n_{2}\right)\right\}= \begin{cases}\sigma_{s}^{2}, & n_{1}=n_{2} \text { or } n_{1}=n_{2} \pm N \\
0, & \text { otherwise }\end{cases} \\
& n_{1}, n_{2} \in\left\{0,1, \ldots, N+N_{\mathrm{cp}}-1\right\}
\end{aligned}
$$

where $\sigma_{s}^{2} \triangleq E\left\{|s(n)|^{2}\right\}$. As a result, the correlation between $r(n)$ and $r(n+N)$ is equivalent to $E\left\{r(n) r^{*}(n+N)\right\}=$ $E\{a(n)\}=e^{-j 2 \pi \varepsilon} \sum_{l=0}^{L}|h(l)|^{2} E\left\{s(n-l-\theta) s^{*}(n+N-\right.$ $l-\theta)\}$ and has the following property:

$$
\begin{aligned}
& E\left\{r(n) r^{*}(n+N)\right\} \\
& =E\{a(n)\} \\
& = \begin{cases}\sigma_{x}^{2} e^{-j 2 \pi \varepsilon}, & n \in\left\{\theta+L, \theta+L+1, \ldots, \theta+N_{\mathrm{cp}}-1\right\} \\
P_{1}(n) e^{-j 2 \pi \varepsilon}, & n \in \Omega_{1} \\
P_{2}(n) e^{-j 2 \pi \varepsilon}, & n \in \Omega_{2} \\
0, & \text { otherwise }\end{cases}
\end{aligned}
$$

where $\sigma_{x}^{2} \triangleq E\left\{|r(n)|^{2}\right\}-\sigma_{n}^{2}=\sum_{l=0}^{L}|h(l)|^{2} \sigma_{s}^{2}$, and

$$
\begin{aligned}
P_{1}(n) & =\sum_{l=0}^{n-\theta}|h(l)|^{2} \sigma_{s}^{2} \\
\Omega_{1} & \triangleq\{\theta, \theta+1, \ldots, \theta+L-1\} \\
P_{2}(n) & =\sum_{l=n-\theta-N_{\mathrm{cp}}+1}^{L}|h(l)|^{2} \sigma_{s}^{2} \\
\Omega_{2} & \triangleq\left\{\theta+N_{\mathrm{cp}}, \theta+N_{\mathrm{cp}}+1, \ldots, \theta+N_{\mathrm{cp}}+L-1\right\} .
\end{aligned}
$$

Obviously, $P_{1}(n)$ and $P_{2}(n)$ are positive, with $P_{1}(n)<\sigma_{x}^{2}$ and $P_{2}(n)<\sigma_{x}^{2}$. 
A new timing function is now proposed as follows:

$$
\begin{aligned}
& \Lambda(k, m) \triangleq\left|\sum_{n=m}^{N_{\mathrm{cp}}-1} E\left\{r(n+k) r^{*}(n+N+k)\right\}\right| \\
&-\frac{\rho^{2}}{2} \sum_{n=m}^{N_{\mathrm{cp}}-1}\left[E\left\{|r(n+k)|^{2}\right\}\right. \\
&\left.+E\left\{|r(n+N+k)|^{2}\right\}\right] \\
& k \in\left\{0,1, \ldots, N+N_{\mathrm{cp}}-1\right\} ; \quad m \in\left\{0,1, \ldots, N_{\mathrm{cp}}-1\right\}
\end{aligned}
$$

where $\rho \triangleq\left(E\left\{|r(n)|^{2}\right\}-E\left\{|w(n)|^{2}\right\}\right) / E\left\{|r(n)|^{2}\right\}=\sigma_{x}^{2} /$ $\left(\sigma_{x}^{2}+\sigma_{n}^{2}\right)$. From the definition of $\Lambda(k, m)$, we have the following property.

Property 1: The maximum of the function $\Lambda(k, m)$ occurs at the point $(k=\theta, m=L)$ when $\sigma_{x}^{2} \gg \sigma_{n}^{2}$ (high SNR) and $\sigma_{n}^{2} \neq 0$.

Proof: Since $E\left\{|r(n)|^{2}\right\}=\sigma_{x}^{2}+\sigma_{n}^{2}$, the function $\Lambda(k, m)$ is equivalent to

$$
\begin{aligned}
\Lambda(k, m)=\left|\sum_{n=m}^{N_{\mathrm{cp}}-1} E\left\{r(n+k) r^{*}(n+N+k)\right\}\right| \\
-\left(N_{\mathrm{cp}}-m\right) \sigma_{x}^{4} /\left(\sigma_{x}^{2}+\sigma_{n}^{2}\right) .
\end{aligned}
$$

When $\sigma_{x}^{2} \gg \sigma_{n}^{2}$, the second term in (10) can be approximated as $\left(N_{\mathrm{cp}}-m\right) \sigma_{x}^{2}$.

When $k \neq \theta$, it follows from (6) and (10) that the function $\Lambda(k, m)$ satisfies

$$
\begin{aligned}
\Lambda(k \neq \theta, m<L) & <\left(N_{\mathrm{cp}}-m\right) \sigma_{x}^{2}-\left(N_{\mathrm{cp}}-m\right) \sigma_{x}^{2}=0 \\
\Lambda(k \neq \theta, m=L) & <\left(N_{\mathrm{cp}}-L\right) \sigma_{x}^{2}-\left(N_{\mathrm{cp}}-L\right) \sigma_{x}^{2}=0 \\
\Lambda(k \neq \theta, m>L) & \leq\left(N_{\mathrm{cp}}-m\right) \sigma_{x}^{2}-\left(N_{\mathrm{cp}}-m\right) \sigma_{x}^{4} /\left(\sigma_{x}^{2}+\sigma_{n}^{2}\right) \\
& =\left(N_{\mathrm{cp}}-m\right) \Delta\left(\sigma_{x}^{2}, \sigma_{n}^{2}\right)
\end{aligned}
$$

where $\Delta\left(\sigma_{x}^{2}, \sigma_{n}^{2}\right)=\sigma_{x}^{2} \sigma_{n}^{2} /\left(\sigma_{x}^{2}+\sigma_{n}^{2}\right)$.

Similarly, when $k=\theta$, it yields

$$
\begin{aligned}
\Lambda(k=\theta, m<L) & <\left(N_{\mathrm{cp}}-m\right) \sigma_{x}^{2}-\left(N_{\mathrm{cp}}-m\right) \sigma_{x}^{2}=0 \\
\Lambda(k=\theta, m=L) & =\left(N_{\mathrm{cp}}-L\right) \sigma_{x}^{2}-\left(N_{\mathrm{cp}}-L\right) \sigma_{x}^{4} /\left(\sigma_{x}^{2}+\sigma_{n}^{2}\right) \\
& =\left(N_{\mathrm{cp}}-L\right) \Delta\left(\sigma_{x}^{2}, \sigma_{n}^{2}\right) \\
\Lambda(k=\theta, m>L) & =\left(N_{\mathrm{cp}}-m\right) \sigma_{x}^{2}-\left(N_{\mathrm{cp}}-m\right) \sigma_{x}^{4} /\left(\sigma_{x}^{2}+\sigma_{n}^{2}\right) \\
& =\left(N_{\mathrm{cp}}-m\right) \Delta\left(\sigma_{x}^{2}, \sigma_{n}^{2}\right) .
\end{aligned}
$$

Since $\sigma_{n}^{2} \neq 0$, which means that $\Delta\left(\sigma_{x}^{2}, \sigma_{n}^{2}\right)>0,\left(N_{\mathrm{cp}}-\right.$ $L) \Delta\left(\sigma_{x}^{2}, \sigma_{n}^{2}\right)$ in (15) is greater than zero and $\left(N_{\mathrm{cp}}-\right.$ $L) \Delta\left(\sigma_{x}^{2}, \sigma_{n}^{2}\right)$ in (15) is also greater than $\left(N_{\mathrm{cp}}-m\right) \Delta\left(\sigma_{x}^{2}, \sigma_{n}^{2}\right)$ in (13) and (16), where $m>L$. Consequently, it can be concluded from (11)-(16) that the function $\Lambda(k, m)$ achieves its maximum at the point $(k=\theta, m=L)$, and the proof follows.
Based on Property 1, the timing offset $\theta$ can be estimated from the maximum point of the timing function $\Lambda(k, m)$ as

$$
\begin{aligned}
& \hat{\theta}=\arg \max _{k} \max _{m} \Lambda(k, m) \\
& m \in\left\{0,1, \ldots, N_{\mathrm{cp}}-1\right\} ; \quad k \in\left\{0,1, \ldots, N+N_{\mathrm{cp}}-1\right\} .
\end{aligned}
$$

The implementation of this symbol synchronization algorithm is then summarized as follows: 1) Compute the timing function $\Lambda(k, m)$ for $\left\{k \in\left\{0,1, \ldots, N+N_{\mathrm{cp}}-1\right\}, m \in\right.$ $\left.\left\{0,1, \ldots, N_{\mathrm{cp}}-1\right\}\right\}$, and 2) choose $k$ corresponding to the maximum of $\Lambda(k, m)$ as the estimated timing offset. Notice that two parameters (i.e., $k$ and $m$ ) are included in the proposed timing function $\Lambda(k, m)$. This is different from the existing CP-based algorithms [8]-[12], where the timing functions have only one parameter. The parameter $k$ corresponding to the maximum of $\Lambda(k, m)$ gives an estimation of the timing offset. Meanwhile, the parameter $m$ corresponding to the maximum of $\Lambda(k, m)$ also yields an estimation of the channel length $L$. Since channel length estimation is outside the scope of this paper, it will not be further discussed here. On the other hand, it is noticed from the definition of the timing function (9) that channel information (including channel power profile and channel length) is unnecessary here, and there is no restriction on the strength of the first path channel response $h(0)$.

Remark 1: In the existing CP-based algorithms [8], [10], [12], due to the existence of ISI, the maximum of the timing function fluctuates in the range of $[\theta, \theta+L]$, where $L$ is the frequency-selective fading channel length, and the maximum depends on channel conditions. This position uncertainty in the maximum will cause significant fluctuation of the estimated timing offset. Unlike the existing algorithms, the maximum of the proposed timing function $\Lambda(k, m)$ necessarily occurs at the point $(k=\theta, m=L)$. That is, the maximum of $\Lambda(k, m)$ necessarily points to the correct timing offset $\theta$, irrespective of channel conditions. Since the position uncertainty is removed, the fluctuation of the estimated timing offset is mitigated, which means that an accurate estimation is obtained.

Remark 2: From Property 1, it is apparent that the maximum value of $\Lambda(k, m)$ is $\left(N_{\mathrm{cp}}-L\right) \Delta\left(\sigma_{x}^{2}, \sigma_{n}^{2}\right)(15)$, while the value of $\Lambda(k, m)$ at $m=L+1$ may be $\left(N_{\mathrm{cp}}-L-1\right) \Delta\left(\sigma_{x}^{2}, \sigma_{n}^{2}\right)$ (13), (16). They differ only by a factor of $\Delta\left(\sigma_{x}^{2}, \sigma_{n}^{2}\right)$. In the finite-sample scenario, the position of the maximum might be perturbed, which would degrade the estimation performance. To avoid this perturbation, a high-resolution analog-to-digital converter is thus required in practice.

Apparently, from the timing function in (9), $N_{\mathrm{cp}}$ correlation windows are included by means of the parameter $m$, and the timing offset is estimated through a 2-D search. The number of complex multiplications required here is therefore $3 \sum_{i=1}^{N_{\mathrm{cp}}} i=$ $3 N_{\mathrm{cp}}\left(N_{\mathrm{cp}}+1\right) / 2$, while that required in the ML algorithm in [8] and the correlation derivative algorithm in [10] is $3 N_{\mathrm{cp}}$ and $N_{\mathrm{cp}}$, respectively. That is, the complexity of the proposed algorithm in terms of the number of complex multiplications is respectively $\left(N_{\mathrm{cp}}+1\right) / 2$ and $3\left(N_{\mathrm{cp}}+1\right) / 2$ times that of [8] 


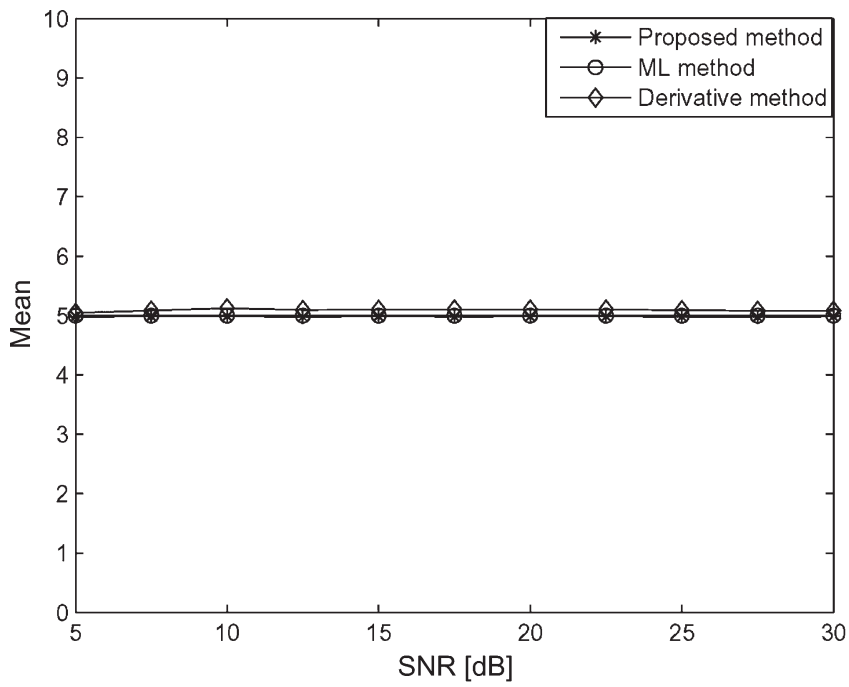

(a)

Fig. 1. Performance versus SNR for flat-fading channels $(L=0)$.

and [10]. On the other hand, the proposed algorithm can work well, irrespective of channel conditions, while the algorithms in [8], [10] do not yield accurate estimation in frequency-selective fading channels. This will be demonstrated by computer simulations in the following section.

\section{Simulation Results}

Computer simulations have been conducted to investigate the performance of the proposed algorithm. In the simulations, an OFDM system with 128 subcarriers $(N=128)$ and $12.5 \%$ CP $\left(N_{\mathrm{cp}}=16\right)$ was considered. The carrier frequency offset was assumed to be one-third of the subcarrier spacing $(\varepsilon=$ $1 / 3)$. The timing offset was set to five sample periods $(\theta=5)$. The performance was evaluated in terms of the mean of the estimated timing offset and the mean square of the estimation error (MSE). All these performance measures were computed by averaging the results over 1000 Monte Carlo realizations. In each run, 1) a data packet with 20 random OFDM symbols was transmitted; 2) all transmitted signals were modulated by the quaternary phase-shift keying scheme; and 3) slow fading was considered here, and the channel responses were randomly generated and time invariant during each run. SNR was defined as

$$
\mathrm{SNR} \triangleq E\left\{|r(n)-w(n)|^{2}\right\} / E\left\{|w(n)|^{2}\right\}=\sigma_{x}^{2} / \sigma_{n}^{2} .
$$

The expectation $E\{\cdot\}$ in $\Lambda(k, m)$ was calculated by averaging the term in brackets over 20 OFDM symbols. With the noise variance $\sigma_{n}^{2}$ estimated by the methods in [11], [14], and [15], the parameter $\rho$ in the proposed algorithm could be computed as $\rho=\left(E\left\{|r(n)|^{2}\right\}-\sigma_{n}^{2}\right) / E\left\{|r(n)|^{2}\right\}$. In the simulation, it was assumed that $\rho$ was perfectly known. For comparison purposes, the ML algorithm in [8] and the correlation derivative algorithm in [10] were also implemented here. Note that in the correlation derivative algorithm [10], the filter length used was set to 16 , and the hard replacement rule was adopted.

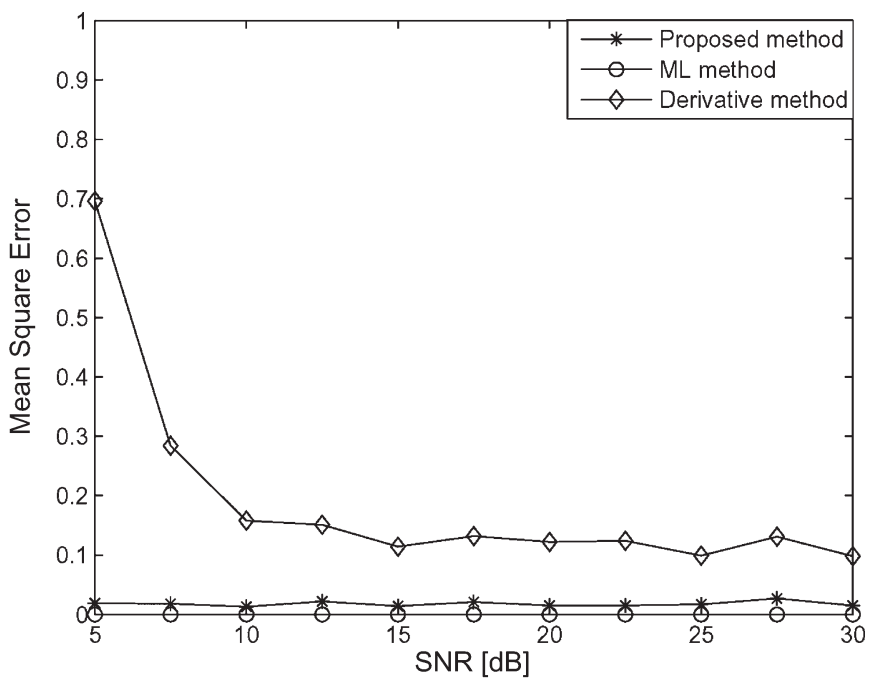

(b)

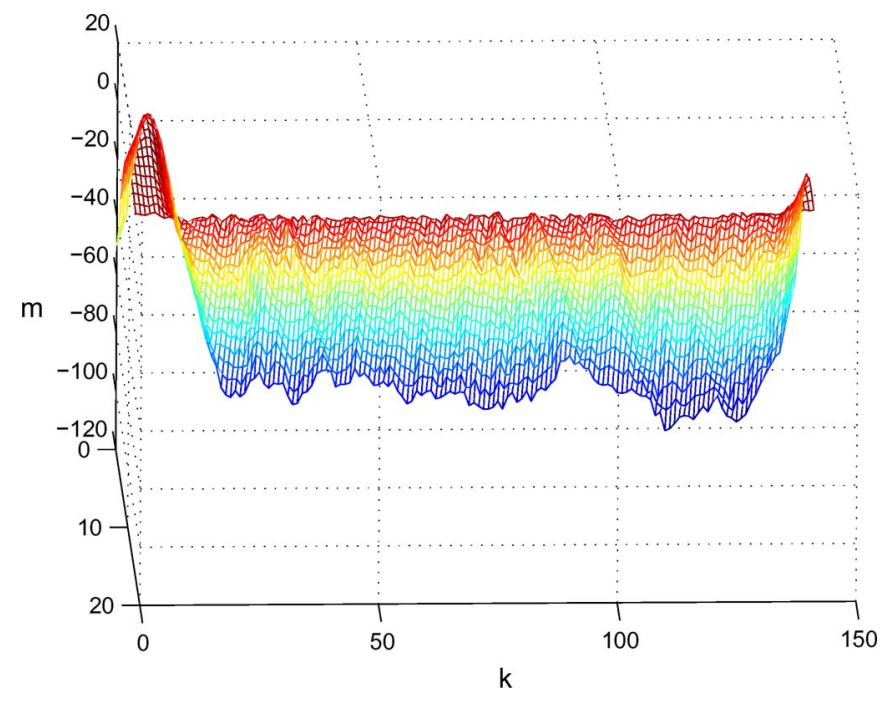

Fig. 2. $\Lambda(k, m)(L=11, \mathrm{SNR}=10 \mathrm{~dB})$.

Flat Rayleigh-fading channels $(L=0)$ were first considered, and the channel response $h(0)$ was randomly generated as a complex Gaussian variable with zero mean and a variance of 2 . Fig. 1(a) and (b) shows the mean and MSE of the timing offset estimated by various algorithms under consideration. It is obvious that all of these algorithms yield an unbiased estimation of the timing offset, while the MSE for the proposed algorithm is lower than that of the derivative algorithm and is close to that of the ML algorithm. Notice that the ML algorithm is the best under flat-fading channels, and its MSE approaches zero since no interference exists in the received signals.

Next, frequency-selective fading channels with 12 independent sample-spaced Rayleigh-fading taps $(L=11)$ were considered. In detail, the channel responses $h(0)-h(11)$ were modeled as independent and complex Gaussian random variables with zero mean and variances of [1.9560, 1.8287, 1.6321, $1.3868,1.1172,0.8481,0.6007,0.3911,0.2281,0.1136$, $0.0434,0.0090]$, respectively. The function $\Lambda(k, m)$ (SNR $=$ $10 \mathrm{~dB}$ ) is first shown in Fig. 2, since it is essential for the 


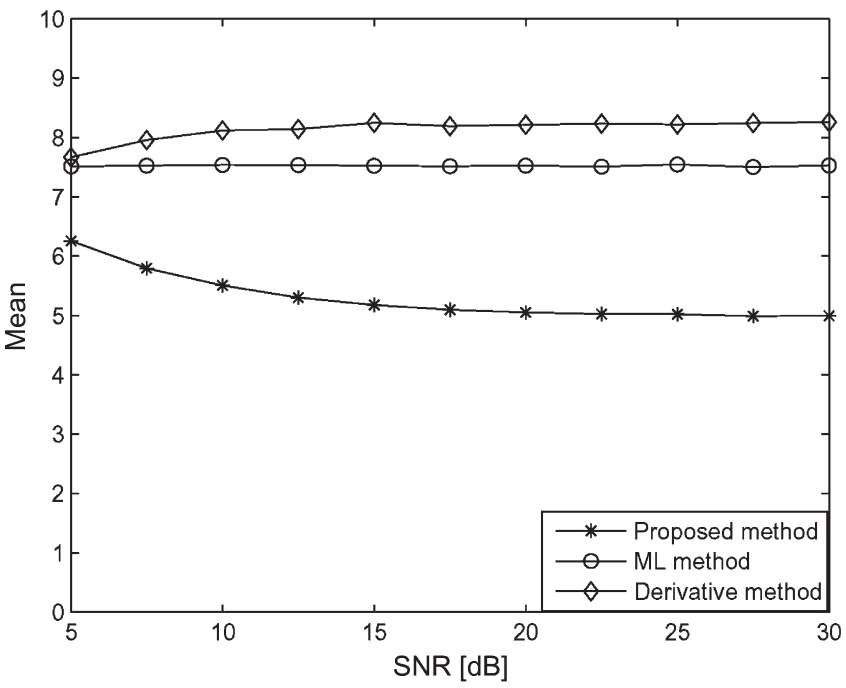

(a)

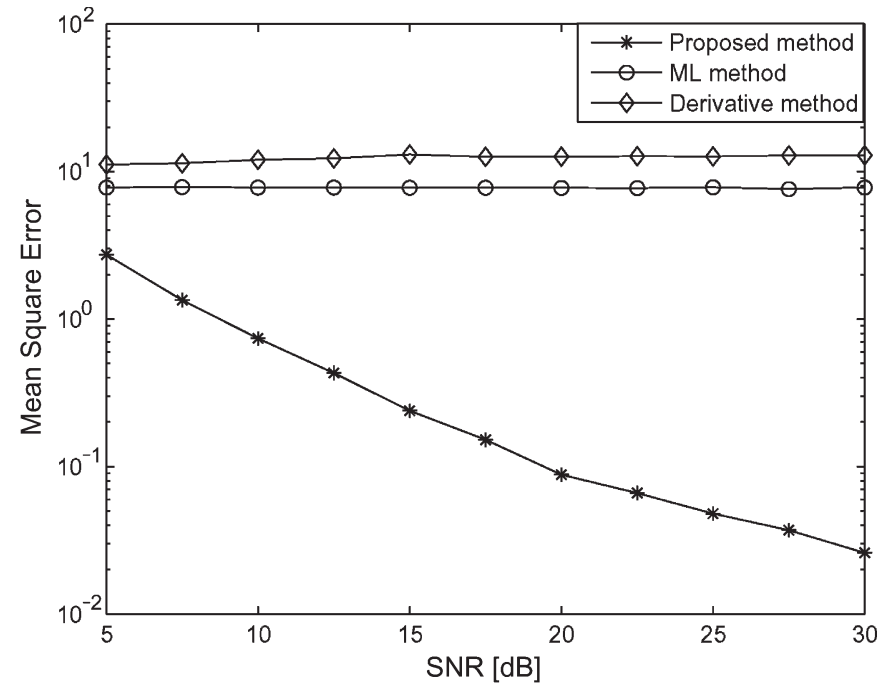

(b)

Fig. 3. Performance versus SNR for frequency-selective fading channels $(L=11)$.

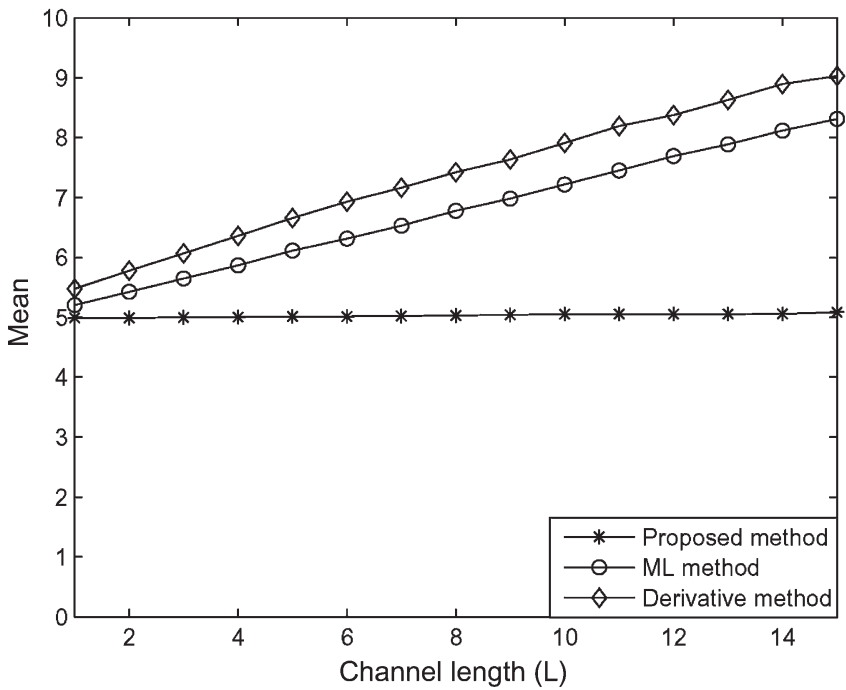

(a)

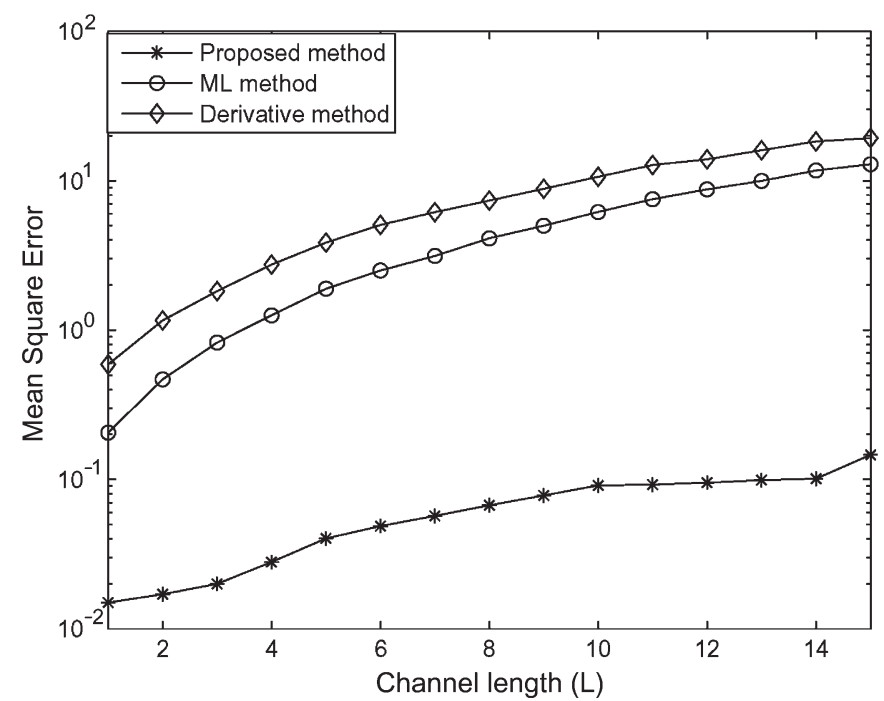

(b)

Fig. 4. Performance versus channel length $(\mathrm{SNR}=20 \mathrm{~dB})$.

derivation of the proposed algorithm. It is shown that the maximum of $\Lambda(k, m)$ points to the correct timing offset $(k=5)$, which clearly verifies Property 1 . The mean of the timing offset estimated by the three aforementioned methods are then illustrated in Fig. 3(a). It shows that the proposed algorithm is still able to achieve an unbiased estimation of the timing offset, while the ML and derivative algorithms cannot. The MSE performance is finally shown in Fig. 3(b). Obviously, the proposed algorithm has a much smaller MSE than the other two algorithms. In addition, the MSE of the proposed algorithm decreases when SNR increases, while there is little variation in that of the other two algorithms. This can be explained as follows: The dominant factor of the MSE in the proposed algorithm is the additive noise as the maximum of $\Lambda(k, m)$ necessarily points to the correct timing offset. On the other hand, the other two algorithms yield a timing offset estimation falling within $\theta$ and $\theta+L$ in frequency-selective fading channels, which mainly depends on channel conditions and is therefore little affected by the SNR.

Finally, the channel length $L$ was varied from 1 to 15 $(\mathrm{SNR}=20 \mathrm{~dB})$ to further test the effect of frequency-selective fading channels on the synchronization algorithms. Results are shown in Fig. 4(a) and (b). Unbiased estimation of the timing offset is achieved by the proposed algorithm irrespective of channel length, whereas the mean of the estimated timing offset in the ML and derivative algorithms increases linearly with the channel length $L$. In terms of MSE, the performance of the proposed algorithm slightly degrades when the channel length increases. However, for any given channel length under consideration, the proposed algorithm significantly outperforms the other two algorithms. These results demonstrate that the proposed algorithm is robust to frequency-selective fading channels and can significantly reduce the MSE compared with the ML and derivative algorithms. 


\section{CONCLUSION}

A CP-based blind symbol synchronization algorithm for OFDM systems has been proposed in this paper. A new timing function in which the maximum necessarily points to the correct timing offset, irrespective of channel conditions, has been designed. The proposed algorithm has overcome fluctuations in the existing CP-based algorithms, particularly when the system is operating under frequency-selective fading channels. Channel information is unnecessary, and there is no restriction on the first path channel response, rendering the proposed algorithm more practical. It has been verified by computer simulations that the proposed algorithm achieves an unbiased estimation and significantly reduces the MSE of the estimated timing offset compared with the existing CP-based algorithms when the system is operating under frequency-selective fading channels.

\section{REFERENCES}

[1] B. Muquet, Z. Wang, G. B. Giannakis, M. de Courville, and P. Duhamel, "Cyclic prefixing or zero padding for wireless multicarrier transmissions?" IEEE Trans. Commun., vol. 50, no. 12, pp. 2136-2148, Dec. 2002.

[2] J. Lei and T. S. Ng, "A consistent OFDM carrier frequency offset estimator based on distinctively spaced pilot tones," IEEE Trans. Wireless Commun., vol. 3, no. 2, pp. 588-599, Mar. 2004.

[3] Y. Mostofi and D. C. Cox, "Mathematical analysis of the impact of timing synchronization errors on the performance of an OFDM system," IEEE Trans. Commun., vol. 54, no. 2, pp. 226-230, Feb. 2006.

[4] B. Ai, Z. X. Yang, C. Y. Pan, J. H. Ge, Y. Wang, and Z. Lu, "On the synchronization techniques for wireless OFDM systems," IEEE Trans. Broadcasting, vol. 52, no. 2, pp. 236-244, Jun. 2006.

[5] T. M. Schmidl and D. C. Cox, "Robust frequency and timing synchronization for OFDM," IEEE Trans. Commun., vol. 45, no. 12, pp. 1613-1621, Dec. 1997.

[6] H. Minn, V. K. Bhargava, and K. B. Letaief, "A robust timing and frequency synchronization for OFDM systems," IEEE Trans. Wireless Commun., vol. 2, no. 4, pp. 822-839, Jul. 2003.

[7] S. H. Muller-Weinfurtner, "On the optimality of metrics for coarse frame synchronization in OFDM: A comparison," in Proc. of Ninth IEEE Int. Symp. Personal, Indoor, Mobile Radio Commun., Sep. 1998, pp. 533-537.

[8] J. J. van de Beek, M. Sandell, and P. O. Borjesson, "ML estimation of time and frequency offset in OFDM systems," IEEE Trans. Signal Process., vol. 45 , no. 7, pp. 1800-1805, Jul. 1997.

[9] A. Palin and J. Rinne, "Enhanced symbol synchronization method for OFDM systems in SFN channels," in Proc. IEEE GLOBECOM, Nov. 1998, pp. 2788-2793.

[10] C. Williams, M. A. Beach, and S. McLaughlin, "Robust OFDM timing synchronisation," in Proc. IEEE Veh. Technol. Conf., May 2006, pp. 1947-1950.

[11] R. Negi and J. M. Cioffi, "Blind OFDM symbol synchronization in ISI channels," IEEE Trans. Commun., vol. 50, no. 9, pp. 1525-1534, Sep. 2002.

[12] D. Lee and K. Cheun, "Coarse symbol synchronization algorithms for OFDM systems in multipath channels," IEEE Commun. Lett., vol. 6, no. 10 , pp. 446-448, Oct. 2002.

[13] X. Y. Pan, Y. Q. Zhou, S. D. Ma, and T. S. Ng, "An improved derivative method for symbol synchronization in OFDM systems," in Proc. IEEE WCNC, Mar. 2007, pp. 2463-2467.

[14] T. Cui and C. Tellambura, "Power delay profile and noise variance estimation for OFDM," IEEE Commun. Lett., vol. 10, no. 1, pp. 25-27, Jan. 2006.

[15] X. Xu, Y. Jing, and X. Yu, "Subspace-based noise variance and SNR estimation for OFDM systems," in Proc. IEEE WCNC, Mar. 2005, pp. 23-26.

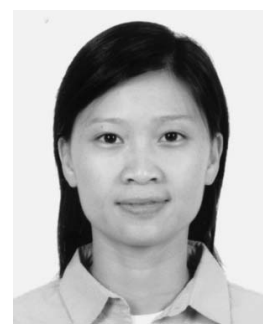

Shaodan Ma received the B.Sc.(Eng.) and the M.Eng.Sc. degrees in electrical engineering from NanKai University, Tianjin, China, in 1999 and 2002, respectively, and the Ph.D. degree in electrical and electronic engineering from The University of Hong Kong, Pokfulam, Hong Kong, in 2006.

Since 2006, she has been with the Department of Electrical and Electronic Engineering, The University of Hong Kong, as a Postdoctoral Fellow. Her research interests include wireless communication systems, spread-spectrum techniques, multipleinput-multiple output systems, orthogonal frequency division multiplexing techniques, and related digital signal processing techniques.

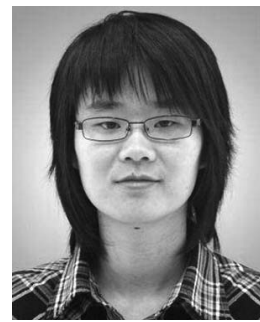

Xinyue Pan received the B.S. degree in electronic science and engineering from Nanjing University, Nanjing, China, in 2004, the M.Sc. degree in communications and signal processing from Imperial College, London, U.K., in September 2005, and the M.Phil. degree in wireless communications from The University of Hong Kong, Pokfulam, Hong Kong.

Her research interests include spread-spectrum, orthogonal frequency division multiplexing, orthogonal frequency division multiple access, symbol synchronization, and other transmission techniques in wireless communications.

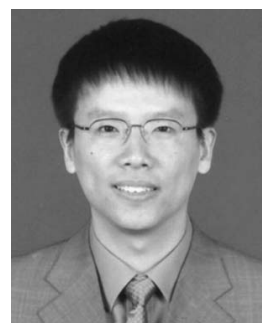

Guang-Hua Yang received the B.S. and M.Eng. degrees in electronic engineering from Nankai University, Tianjin, China, in 1999 and 2002, respectively, and the Ph.D. degree from The University of Hong Kong, Pokfulam, Hong Kong, in 2006.

$\mathrm{He}$ is currently a Postdoctoral Fellow with The University of Hong Kong. His research area includes wireless communications and networking, multimedia computing, and signal processing.

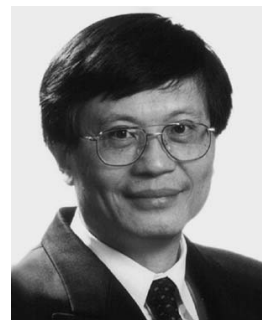

Tung-Sang Ng (F'03) received the B.Sc.(Eng.) degree from The University of Hong Kong, Pokfulam, Hong Kong, in 1972 and the M.Eng.Sc. and $\mathrm{Ph} . \mathrm{D}$. degrees from the University of Newcastle, Callaghan, Australia, in 1974 and 1977, respectively, all in electrical engineering.

$\mathrm{He}$ was with BHP Steel International and the University of Wollongong, Wollongong, Australia, after graduation for 14 years before he returned to The University of Hong Kong in 1991 to be a Professor and the Chair of the Department of Electronic Engineering. He was the Head of the Department of Electrical and Electronic Engineering from 2000 to 2003 and the Dean of Engineering from 2003 to 2007. His current research interests include wireless communication systems, spread-spectrum techniques, code-division multiple access, and digital signal processing. He has authored over 250 papers published in international journals and conference proceedings.

Dr. $\mathrm{Ng}$ is a Fellow of the Institution of Electrical Engineers (IEE) and the Hong Kong Institution of Engineers. He was the General Chair of the 1997 IEEE International Symposium on Circuits and Systems and the Vice President-Region 10 of the IEEE Circuits and Systems Society in 1999 and 2000. He was an Executive Committee Member and a Board Member of the IEE Informatics Divisional Board (1999-2001) and was a Member of the IEE Council (1999-2001). He received the Honorary Doctor of Engineering degree from the University of Newcastle in 1997, the Senior Croucher Foundation Fellowship in 1999, the IEEE Third Millenium Medal in 2000, and the Outstanding Researcher Award from The University of Hong Kong in 2003. 